\title{
Vertical Transmission of SARS-CoV-2 (COVID-19): Are Hypotheses More than Evidences?
}

\author{
Cinzia Auriti, MD ${ }^{1}$ Domenico Umberto De Rose, MD ${ }^{1}$ Chryssoula Tzialla, MD ${ }^{2}$ Leonardo Caforio, MD \\ Matilde Ciccia, MD ${ }^{4}$ Paolo Manzoni, MD, $\mathrm{PhD}^{5}$ Mauro Stronati, MD²
}

\footnotetext{
1 Neonatal Intensive Care Unit, Department of Medical and Surgical Neonatology, “Bambino Gesù” Children's Hospital IRCCS, Rome, Italy

2 Neonatal Intensive Care Unit, IRCCS Foundation Policlinico "San Matteo," Pavia, Italy

${ }^{3}$ Fetal Medicine and Surgery Unit, Department of Medical and Surgical Neonatology, “Bambino Gesù” Children's Hospital IRCCS, Rome, Italy

${ }^{4}$ Neonatal Intensive Care Unit, Department of Women's and Children's Health, Maggiore Hospital, Bologna, Italy

${ }^{5}$ Department of Pediatrics and Neonatology, University Hospital Degli Infermi, Biella, Italy
}

Address for correspondence Cinzia Auriti, MD, Neonatal Intensive Care Unit, Department of Medical and Surgical Neonatology, “Bambino Gesù” Children's Hospital IRCCS, Piazza S. Onofrio 4, 00165 Rome, Italy (e-mail: cinzia.auriti@opbg.net).

Am J Perinatol 2020;37(suppl S2):S31-S38.

\begin{abstract}
Keywords

- newborns

- COVID-19

- infants

- SARS-CoV-2

- coronavirus

- pregnancy

In spite of the increasing, accumulating knowledge on the novel pandemic coronavirus severe acute respiratory syndrome coronavirus-2 (SARS-CoV-2), questions on the coronavirus disease-2019 (COVID-19) infection transmission from mothers to fetuses or neonates during pregnancy and peripartum period remain pending and have not been addressed so far. SARS-CoV-2, a RNA single-stranded virus, has been detected in the amniotic fluid, in the cord blood and in the placentas of the infected women. In the light of these findings, the theoretical risk of intrauterine infection for fetuses, or of peripartum infection occurring during delivery for neonates, has a biological plausibility. The extent of this putative risk might, however, vary during the different stages of pregnancy, owing to several variables (physiological modifications of the placenta, virus receptors' expression, or delivery route). This brief review provides an overview of the current evidence in this area. Further data, based on national and international multicenter registries, are needed not only to clearly assess the extent of the risk for vertical transmission, but also to ultimately establish solid guidelines and consistent recommendations.
\end{abstract}

\section{Key Points}

- Questions on the COVID-19 infection transmission from mothers to fetuses or neonates during pregnancy and peripartum period remain pending so far.

- The theoretical risk of intrauterine infection for fetuses, or of neonatal infection during delivery for neonates, has a biological plausibility.

- A caution is recommended in the interpretation of clinical and laboratory data in neonates.

Novel coronavirus severe acute respiratory syndrome coronavirus-2 (SARS-CoV-2) and its pandemic disease (coronavirus disease-2019 [COVID-19]) led the medical community to face the risk of infection by a virus with new characteristics of pathogenicity and diffusion. In the area of maternal- fetal-neonatal medicine, many questions have arisen. Among them are: how to manage neonates tested positive for SARS-COV-2, how to manage mothers with COVID-19 due for delivery and neonatal care, whether there is a maternalfetal transmission of the virus, whether to allow
Copyright $\odot 2020$ by Thieme Medical Publishers, Inc., 333 Seventh Avenue, New York, NY 10001, USA. Tel: +1(212) 760-0888. ISSN 0735-1631. 
breastfeeding or not, and what can be the preventive measures to be adopted in the neonatology wards.

Despite the publication of at least 21,167 scientific articles on the subject so far available on PubMed at the time of writing this article, a wide number of pending issues related to the biology of the virus, its pathogenesis and the pathophysiology of the infection-as well as treatment options-remain unaddressed.

SARS CoV-2 is a single stranded RNA virus, with different structural proteins. Transmission occurs primarily via droplets within a range of approximately $1.8 \mathrm{~m}$ (6 feet), ${ }^{1}$ but other transmission routes could add, since the virus has been found in many biological liquids (saliva, feces, and blood) and at least $1 \%$ of the adult population has a transient viremia with a low level of viral copies. ${ }^{2}$

The risk of fetal infection due to maternal-fetal transmission of the virus is one of the most debated aspects, implying how infection in neonates could be prevented in the peripartum period.

With the aim to address this area, we performed a literature search using key electronic database PubMed. A list of search terms and phrases was compiled to focus on the general topics of "neonates" or "newborns" or "vertical transmission" or "maternal-fetal transmission" or "pregnancy" or "pregnant women" and "COVID-19" or "SARS-CoV-2," without imposing restrictions on date or year, locations, study design, study aim, or inclusion/exclusion criteria. We reviewed articles published until June 2, 2021 ( $n=2,256)$. Studies not written in English or Italian were withdrawn $(n=86)$. The potentially relevant articles were fully evaluated by two reviewers (C.A. and D.U. D.R.). Case reports on neonates who were discharged home and then readmitted were excluded.

\section{Positive Maternal-Infant Dyads}

The hypothesis that the virus may be present in the amniotic fluid, in the cord blood, and in the placenta during maternal infection in pregnancy, consequently infecting the fetus or the neonate at delivery, has a biological plausibility. Nonetheless a caution is recommended in the interpretation of clinical and laboratory data.

The first data from China related to maternal-infant dyads tested with reverse transcriptase-polymerase chain reaction (RT-PCR), performed on nasopharyngeal exudate samples, suggested that intrauterine transmission of the virus was unlikely, although not impossible. ${ }^{3,4}$ It should be noted that the sensitivity of the RT-PCR is 63\% in the nasal swab, 93\% in the bronchoalveolar lavage liquid, and $29 \%$ in the feces of infected subjects. ${ }^{2}$ In addition, a single nasopharyngeal swab could not be sufficient for diagnosis, but the samples should be taken from more than one site to improve diagnostic accuracy and reduce the number of false negatives. ${ }^{5}$ Chen et al first reported the data of a retrospective cohort of nine pregnant women with an ongoing infection, in which the virus was searched by RT-PCR in the amniotic fluid, in the umbilical cord, in the nasopharyngeal exudate of six of the neonates, and in breast milk, with always negative results. $^{3}$ At the Policlinico "San Matteo" in Pavia (Italy), 15 pregnant women (among them, five were symptomatic) tested positive for SARS-CoV-2, but no neonates had positive nasopharyngeal swabs at birth and at 5 days of life, despite infants were breastfed and shared the room with their mothers [unpublished data].

To date, it is unclear whether the vertical transmission of SARS-CoV-2 from mother to fetus occurs or not. The currently published literature describes only infants with positive nasopharyngeal swab after birth, which could be related to the horizontal transmission immediately in postpartum, by the contact with infected mothers or with positive health care personnel providing care at the time of delivery. -Table 1 summarizes the main publications supporting the possibility of a maternal-fetal transmission of SARSCoV-2 virus: most authors described no positive cord blood samples. Costa et al reported no maternal-fetal transmission in an infant, although cord blood and placental tissue samples tested positive. ${ }^{6}-$ Table 2 shows the main articles which exclude or consider insufficient data available to support the maternal-fetal transmission of SARS-CoV-2 virus during the first week of life.

\section{SARS-CoV-2 and Serological Tests}

New questions have arisen with the implementation of tests for antibodies detection in serum, ${ }^{7}$ available only from February 2020. The presence of specific immunoglobulin $\mathrm{M}$ (IgM) antibodies against SARS-CoV-2 in the cord blood of few hours-old neonates, described by some authors, has generated some concern. ${ }^{8,9}$

High values of specific IgM at 2 hours of life were reported in a neonate born from caesarean section, who at birth was immediately separated from the mother (she was positive since 23 days before delivery) and isolated. However, the nasopharyngeal swab of this neonate was always negative in five samples and symptoms of SARS-CoV-2 virus infection never appeared. ${ }^{8}$ Zeng et al described six additional infants from six women with COVID-19, diagnosed on the basis of clinical, radiological symptoms, and positive nasopharyngeal swab. These infants underwent blood sampling, RT-PCR nasopharyngeal swab, serum immunoglobulin G (IgG), and IgM assay immediately after birth. All neonates tested negative for RT-PCR-SARS-CoV-2 on blood and nasopharyngeal exudate. Three among these six infants showed elevated SARS-CoV-2 specific IgG and IgM levels, with their mothers having positive IgG and IgM values, while the remaining three showed high IgG but not IgM antibodies, whereas mothers tested positive for $\operatorname{IgG}$ but not for $\operatorname{IgM}{ }^{9}$

The fetus produces IgG and IgM antibodies approximatively since the 20th week of gestation. As mother's IgG antibodies are continuously transferred to the fetus during pregnancy, most of IgG is of maternal origin. IgM antibodies do not cross the placenta, therefore their presence in the fetus or neonate suggests fetal or neonatal production in response to an intrauterine infection. ${ }^{10}$ However, considering the lack of data about sensitivity and specificity of SARS-CoV-2 antibodies, the interpretation of serological tests in COVID-19 infection diagnosis requires further studies. A cautious awareness about these uncertainties should be maintained to correctly evaluate 


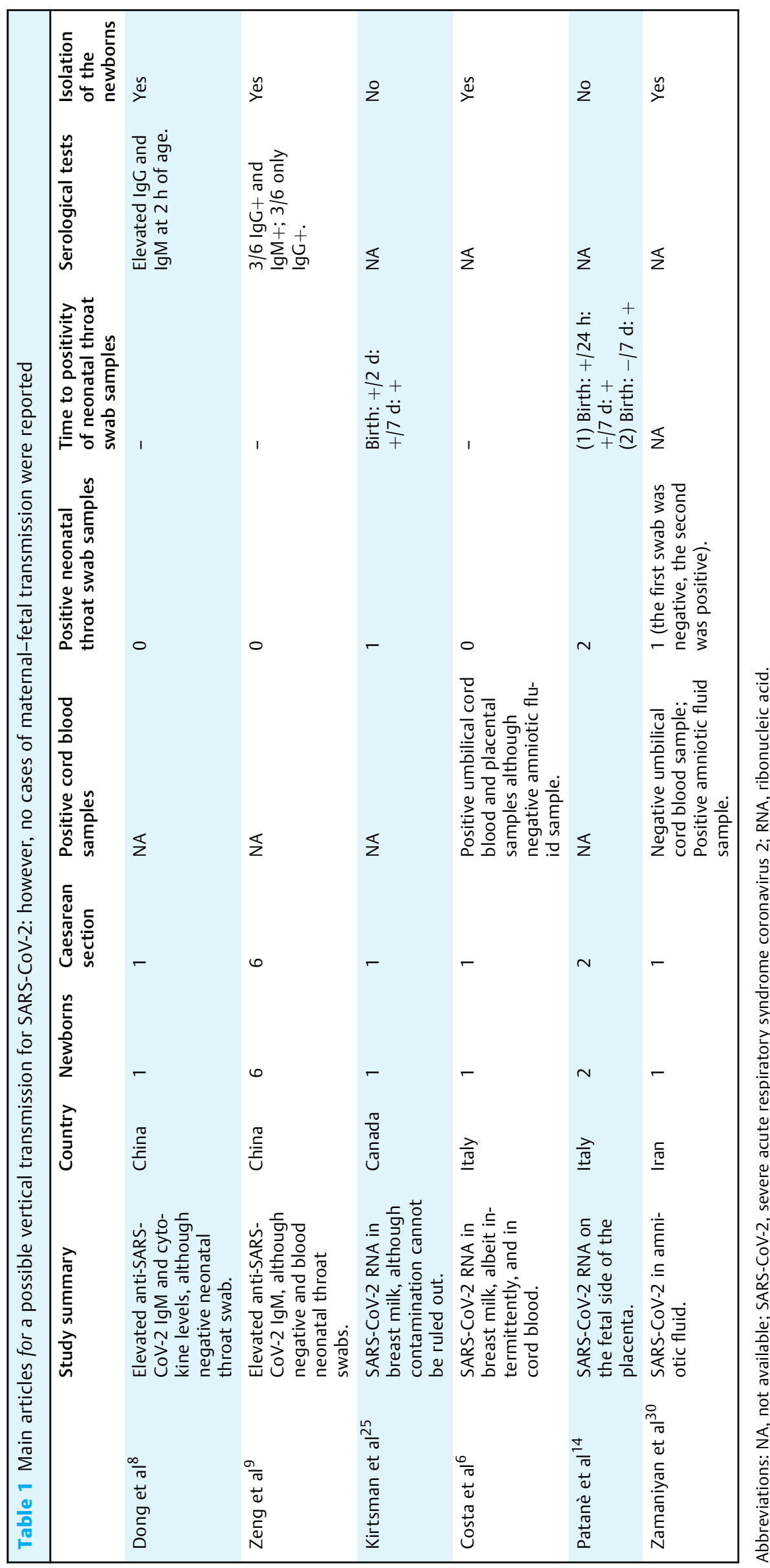




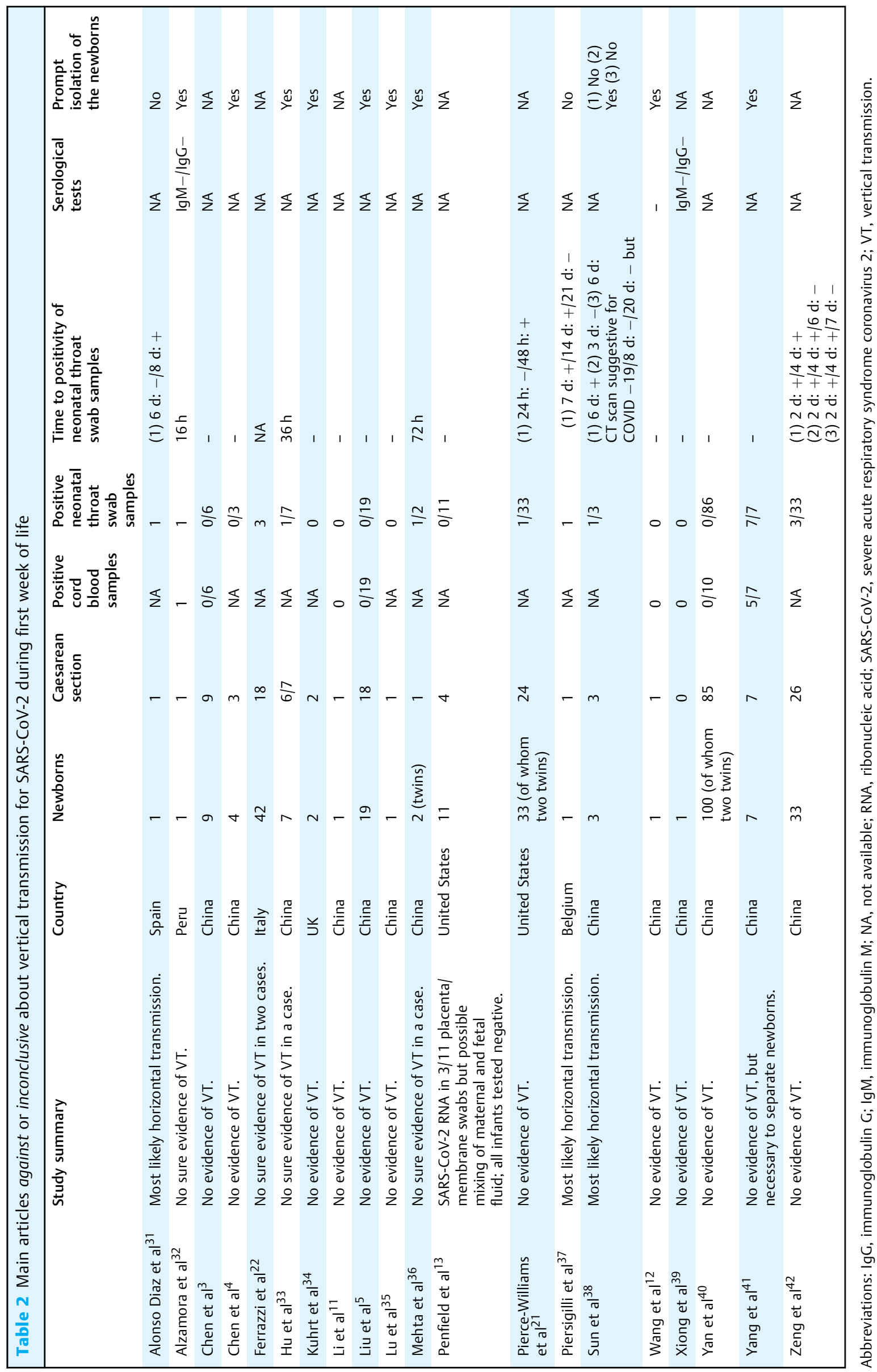


the somewhat contrasting data reported so far about a possible maternal-fetal transmission of SARS-CoV-2.

\section{SARS-CoV-2 and Placenta}

The SARS-CoV-2 virus has never been found so far in the umbilical cord blood of neonates and in the amniotic fluid of most COVID-19 positive pregnant women, ${ }^{3,11,12}$ but testing of all intrauterine tissue samples may be important. To detect viral RNA in the placenta is a crucial point to understand the mechanisms of the maternal-fetal transmission. In the cohort of 32 positive pregnant women described by Penfield et $\mathrm{al}^{13}$ none of their neonates showed symptoms of COVID-19 infection, despite three out of 11 mother's placentas resulted SARSCoV-2 positive by RT-PCR. No infant tested SARS-CoV-2 positive on day one of life through repeated nasopharyngeal swabs. No data are reported on the results of SARS-CoV-2 RT-PCR on blood samples from neonates born to women with positive placental tests. Placental samples were obtained between amnion and chorion after manual separation of the membranes: their positivity supported the theoretical possibility of an intrapartum transmission of the virus to the neonate.

Patanè et al from Bergamo, the epicenter of viral spread in Italy, described 22 symptomatic COVID-19 women, with two neonates tested positive on nasopharyngeal swabs, despite the protective equipment used by the mother and health care workers. ${ }^{14}$ Neonates had only mild symptoms, such as poor feeding. The analysis of the fetal side of these mothers' placentas showed chronic intervillositis, with macrophages CD68+ infiltration, associated with viral RNA in both cases. On the contrary, this histological picture of placental inflammation was not detected in placentas of all COVID-19 positive women who delivered infants with negative swabs. The presence of SARS-CoV-2 RNA in the syncytiothrophoblast supports the possibility of vertical transmission from mother to the baby in utero, but again no data are reported on the results of SARS-CoV-2 RT-PCR on newborns' blood samples.

\section{Perinatal Outcomes and Mechanisms of Infection}

Most case reports described infections contracted by mothers in the last trimester of pregnancy or in the peripartum, when the fetus has matured and many of the physiological phenomena of pregnancy have come to an end. ${ }^{15}$ There are no case reports on infections contracted early in pregnancy, with teratogenic effect on the neonate. No autopsy reports have been published on fetuses aborted during maternal COVID-19 infection in pregnancy.

As in other viral diseases that may complicate pregnancy, the susceptibility to infection could vary according to gestational age, due to the physiological changes in immunehormonal responses and in maternal tissues structure. The risk of transplacental passage of the SARS-CoV-2 virus could therefore vary with the progress of gestation. Another critical factor conditioning viral transmission could be the association of maternal infection with underlying diseases, such as diabetes or other chronic diseases.
One of the most common hypotheses of the infection mechanism considers the receptor of the angiotensin-2 converting enzyme (ACE-2-R) as target of SARS-CoV-2, ${ }^{16}$ to which the virus would attach itself through a structural protein (spike), and in particular through a specific protein site, the receptor binding domain. ACE-2-R is expressed on the membrane of many host cells, in particular of respiratory and intestinal tract (hence the respiratory transmission, and also-as recently described-the possibility of orofecal transmission), ${ }^{17}$ but it is also expressed widely in placenta, uterus, ovaries, and vagina ${ }^{18}$ (-Fig. 1). The placental expression of this receptor seems extremely low between 6 and 14 weeks of pregnancy, which would make unlikely the infection in the first trimester. We also know that, in addition to binding with the ACE- 2 receptor, a transmembrane protease serine type 2 seems essential for the entry and fusion of the virus into the host cell. The expression of this protein in maternal-fetal interface as well as in multiple fetal organs increases as gestational age progresses, as shown by matching different assessments performed at 8 and 24 weeks. This phenomenon could meaningfully add to the list of factors that influence the timing of the infection in pregnancy, by limiting the frequency of early infections. ${ }^{19}$

Angiotensin II and ACE-2 seem to play a role in the development of the follicle and in ovulation, modulating angiogenesis and luteal degeneration, changes in endometrial tissue, and the development of the embryo. Cases of abortion and preterm birth have been described in women infected with SARS-CoV-2 virus. In a large series of 295 SARS-CoV-2 positive women, four abortion cases were reported $(1.4 \%){ }^{20}$ Up to $75 \%$ of infants born to critically positive mothers were premature babies. ${ }^{21}$ Furthermore, approximately $28.3 \%$ of those born to positive mothers required hospitalization in neonatal intensive care unit, for prematurity and/or infection and the observed neonatal mortality was $0.5 \%$ (only one of 221 neonates). ${ }^{20}$ Therefore, the hypothesis of possible negative effects of the COVID-19 infection on the physiological progression of pregnancy at any stage of gestation and childbirth is not unjustified. $^{18}$

\section{Methods of Delivery}

The first published data had mainly reported neonates born via caesarean section (76.8\%), with CS performed to reduce the risk of vertical transmission and decrease putative risks among staff members. ${ }^{15}$ Many infants born via caesarean section could have had a decreased length of exposure to infected tissues, which might be associated with a decreased likelihood of infection. ${ }^{13}$

From the data of a multicentric study performed in 12 hospitals in Northern Italy (Lombardy, Veneto, and EmiliaRomagna), two neonates of the group of vaginal deliveries and one neonate of the group of elective caesarean section, performed for reasons unrelated to COVID-19 infection, tested positive while no positive neonates were observed in the group of elective caesarean sections due to COVID-19. The authors concluded that the risk of intrapartum transmission during vaginal delivery cannot be ruled out, 


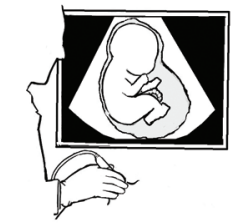

\section{MATERNAL-FETAL TRANSMISSION ?}

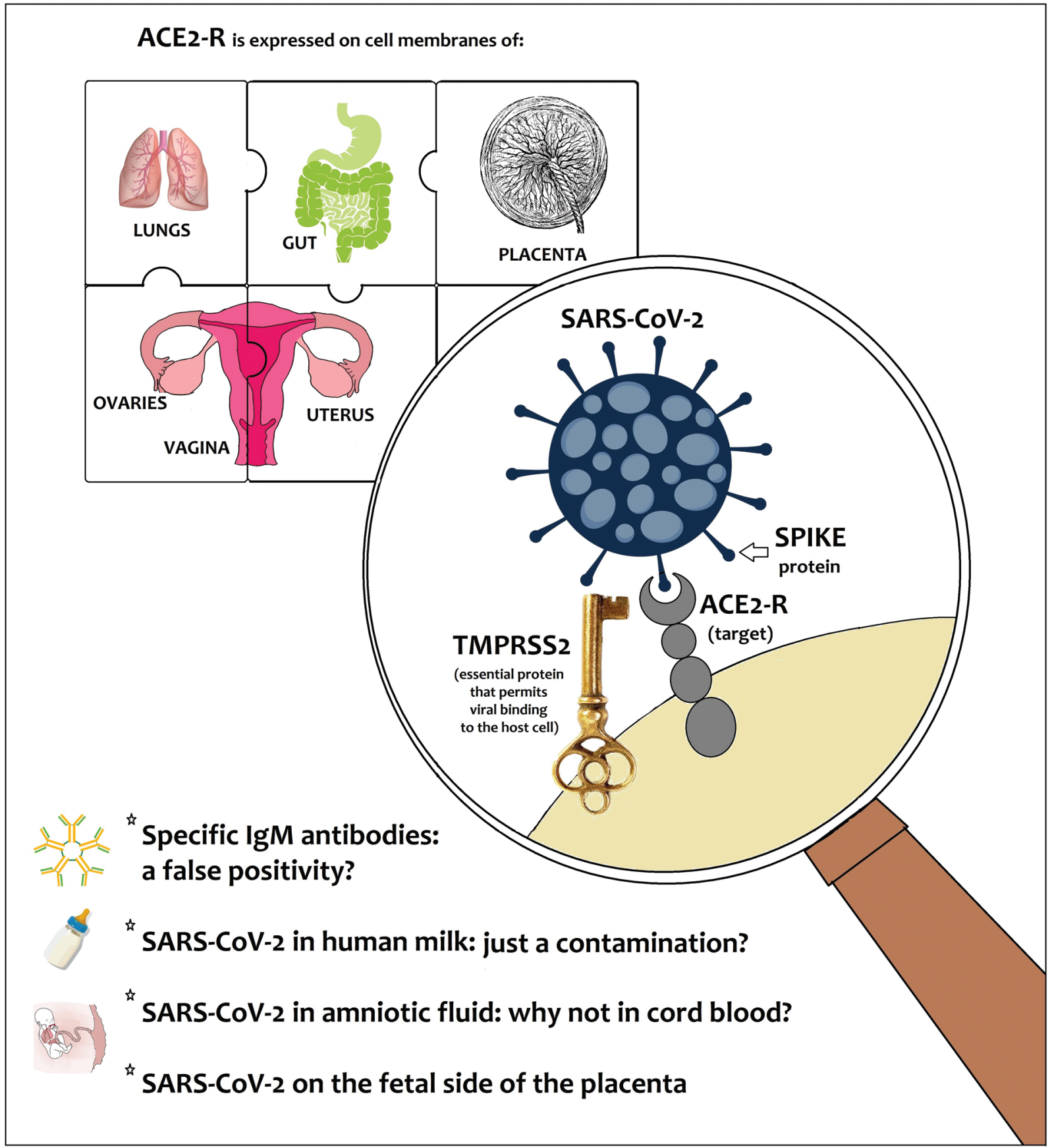

Fig. 1 The difficult-to-solve puzzle of maternal-fetal transmission of SARS-CoV-2 and expression of receptor of the angiotensin-2 converting enzyme (ACE-2-R). SARS-CoV-2, severe acute respiratory syndrome coronavirus 2.

although it is difficult to exclude that positive babies acquired the infection in the postpartum. ${ }^{22}$

The current evidence-based approach, however, is to support vaginal birth in all women, also when tested SARS-CoV-2 positive, if mothers' clinical conditions are good. Caesarean section should be reserved for women affected by COVID-19 featuring severe respiratory morbidity.

\section{Breastfeeding}

Breastfeeding by positive COVID-19 mothers has been since ever a broadly debated topic within the scientific community.

Possible transmission through maternal milk should require not only detection of the virus in fresh breast milk samples, but also actual onset of infection in neonates' breastfed from 
positive mother whose milk has been tested positive for the virus. None of these evidences has been demonstrated so far.

SARS-CoV-2 virus has not been detected in human milk samples in most reports-thus excluding the transmission by this route ${ }^{23,24}$-with the exception of scattered reports describing SARS-CoV-2 RNA detection in maternal milk samples. $^{6,25-27}$ Notably, the same authors stated that the risk of the milk contamination by maternal respiratory secretions could not be ruled out. ${ }^{25}$ Costa et al reported that the excretion of the virus in breastmilk was intermittent: only three of the six analyzed breast-milk samples (50\%) were positive for SARS-CoV-2 RNA. ${ }^{6}$

In the light of the available evidence, the Italian Society of Neonatology recommends commencement of breastfeeding in all women with suspected or confirmed SARS-CoV-2 infection if they are in good clinical conditions. It is also recommended that this option should be discussed with the father, with clear indications of the associated benefits, risks, and correct hygiene rules to be mandatorily applied. ${ }^{28}$

\section{Conclusion}

Further studies on large population samples are needed to fully understand whether maternal-fetal transmission of SARS-CoV-2 virus occurs, and whether vertically acquired neonatal COVID-19 infection occurs.

In this view, initiatives of multicenter registries on infants born to SARS-CoV-2 positive mothers and infants with acquired COVID-19 should be encouraged, such as the national registry promoted by Italian Society of Neonatology and the international "Pregnancy and Neonatal outcomes for women with COVID-19" Registry (PAN-COVID study). ${ }^{29}$

\section{Authors' Contributions}

C.A. and D.U.D.R. conceptualized and designed the review, analyzed the reported cases, drafted the initial manuscript, and reviewed and revised the manuscript. C.T., L.C., M.C., P.M., and M.S reviewed the extracted data and analyzed the cases and reviewed and revised the final manuscript including all tables and the figure. All authors critically reviewed the manuscript for important intellectual content. All authors approved the final manuscript as submitted and agreed to be accountable for all aspects of the work.

\section{Note}

A shorter communication on this topic in Italian has been initially sent to the bulletin of the Italian Society of Neonatology (SIN Informa).

Funding

None.

Conflict of Interest

None declared.

\section{References}

1 Setti L, Passarini F, De Gennaro G, et al. Airborne transmission route of COVID-19: why 2 meters/ 6 feet of inter-personal distance could not be enough. Int J Environ Res Public Health 2020;17(08): E2932

2 Wang W, Xu Y, Gao R, et al. Detection of SARS-CoV-2 in different types of clinical specimens. JAMA 2020;323:1843-1844

3 Chen $\mathrm{H}$, Guo J, Wang C, et al. Clinical characteristics and intrauterine vertical transmission potential of COVID-19 infection in nine pregnant women: a retrospective review of medical records. Lancet 2020;395(10226):809-815

4 Chen Y, Peng H, Wang L, et al. Infants born to mothers with a new coronavirus (COVID-19). Front Pediatr 2020;8:104

5 Liu W, Wang J, Li W, Zhou Z, Liu S, Rong Z. Clinical characteristics of 19 neonates born to mothers with COVID-19. Front Med 2020; 14(02):193-198

6 Costa S, Posteraro B, Marchetti S, et al. Excretion of SARS-CoV-2 in human breastmilk samples. Clin Microbiol Infect 2020 (e-pub ahead of print). Doi: 10.1016/j.cmi.2020.05.027

7 Sethuraman N, Jeremiah SS, Ryo A. Interpreting diagnostic tests for SARS-CoV-2. JAMA 2020;2020:E1-E3

8 Dong L, Tian J, He S, et al. Possible vertical transmission of SARSCoV-2 from an infected mother to her newborn. JAMA 2020; 323:1846-1848

9 Zeng H, Xu C, Fan J, et al. Antibodies in infants born to mothers with COVID-19 pneumonia. JAMA 2020;323:1848-1849

10 Kimberlin DW, Stagno S. Can SARS-CoV-2 infection be acquired in utero? More definitive evidence is needed. JAMA 2020; 323:1788-1789

11 Li Y, Zhao R, Zheng S, et al. Lack of vertical transmission of severe acute respiratory syndrome coronavirus 2, China. Emerg Infect Dis 2020;26(06):1335-1336

12 Wang X, Zhou Z, Zhang J, Zhu F, Tang Y, Shen X. A case of 2019 novel coronavirus in a pregnant woman with preterm delivery. Clin Infect Dis 2020:ciaa200 (e-pub ahead of print). Doi: $10.1093 /$ cid/ciaa200

13 Penfield CA, Brubaker SG, Limaye MA, et al. Detection of SARS-CoV-2 in placental and fetal membrane samples. Am J Obstet Gynecol MFM 2020 (e-pub ahead of print). Doi: 10.1016/j.ajogmf.2020.100133

14 Patanè L, Morotti D, Giunta MR, et al. Vertical transmission of COVID-19: SARS-CoV-2 RNA on the fetal side of the placenta in pregnancies with COVID-19 positive mothers and neonates at birth. Am J Obstet Gynecol MFM 2020;2020:100145

15 De Rose DU, Piersigilli F, Ronchetti MP, et al; Study Group of Neonatal Infectious Diseases of The Italian Society of Neonatology (SIN). Novel coronavirus disease (COVID-19) in newborns and infants: what we know so far. Ital J Pediatr 2020;46(01):56

16 Sanders JM, Monogue ML, Jodlowski TZ, Cutrell JB. Pharmacologic treatments for coronavirus disease 2019 (COVID-19): a review. JAMA 2020;323:1824-1836

17 Yeo C, Kaushal S, Yeo D. Enteric involvement of coronaviruses: is faecal-oral transmission of SARS-CoV-2 possible? Lancet Gastroenterol Hepatol 2020;5(04):335-337

18 Yan J, Li R-Q, Wang H-R, et al. Potential influences of COVID-19/ ACE2 on female reproductive system 2020. OSF Preprints. doi:10.1093/molehr/gaaa030

19 Li M, Chen L, Zhang J, Xiong C, Li X. The SARS-CoV-2 receptor ACE2 expression of maternal-fetal interface and fetal organs by singlecell transcriptome study. PLoS One 2020;15(04):e0230295

20 Juan J, Gil MM, Rong Z, Zhang Y, Yang H, Poon LC. Effects of coronavirus disease 2019 (COVID-19) on maternal, perinatal and neonatal outcomes: a systematic review. Ultrasound Obstet Gynecol 2020. Doi: 10.1002/uog.22088

21 Pierce-Williams RA, Burd J, Felder L, et al. Clinical course of severe and critical COVID-19 in hospitalized pregnancies: a US cohort study. Am J Obstet Gynecol MFM 2020 (e-pub ahead of print). Doi: 10.1016/j.ajogmf.2020.100134

22 Ferrazzi E, Frigerio L, Savasi V, et al. Vaginal delivery in SARS-CoV2 infected pregnant women in Northern Italy: a retrospective analysis. BJOG An Int J Obstet Gynaecol 2020 (e-pub ahead of print). Doi: 10.1111/1471-0528.16278 
23 Salvatori G, De Rose DU, Concato C, et al. Managing COVID-19positive maternal-infant dyads: an Italian experience. Breastfeed Med 2020;15(05):347-348

24 Lackey KA, Pace RM, Williams JE, et al. SARS-CoV-2 and human milk: what is the evidence? Matern Child Nutr 2020 (e-pub ahead of print). Doi: $10.1111 / \mathrm{mcn} .13032$

25 Kirtsman M, Diambomba Y, Poutanen SM, et al. Probable congenital SARS-CoV-2 infection in a neonate born to a woman with active SARS-CoV-2 infection. CMAJ 2020;192(24):E647-E650

26 Tam PCK, Ly KM, Kernich ML, et al. Detectable severe acute respiratory syndrome coronavirus 2 (SARS-CoV-2) in human breast milk of a mildly symptomatic patient with coronavirus disease 2019 (COVID-19). Clin Infect Dis 2020 (e-pub ahead of print). Doi: 10.1093/cid/ciaa673

27 Groß R, Conzelmann C, Müller JA, et al. Detection of SARS-CoV-2 in human breastmilk. Lancet 2020;395(10239):1757-1758

28 Davanzo R, Moro G, Sandri F, Agosti M, Moretti C, Mosca F. Breastfeeding and coronavirus disease-2019: ad interim indications of the Italian Society of Neonatology endorsed by the Union of European Neonatal \& Perinatal Societies. Matern Child Nutr 2020; 16(03): 13010

29 PAN-COVID. Pregnancy and neonatal outcomes for women with COVID-19 Registry (PAN-COVID study). 2020. Available at: https://pan-covid.org/. Accessed June 30

30 Zamaniyan M, Ebadi A, Aghajanpoor Mir S, Rahmani Z, Haghshenas M, Azizi S. Preterm delivery in pregnant woman with critical COVID-19 pneumonia and vertical transmission. Prenat Diagn 2020 (e-pub ahead of print). Doi: 10.1002/pd.5713

31 Alonso Diaz C, Maestro ML, Moral Pumarega MT, Florez Anton B, Pallas Alonso C. First case of neonatal infection due to COVID-19 in Spain. An Pediatr (Engl Ed) 2020;92(04):237-238

32 Alzamora MC, Paredes T, Caceres D, Webb CM, Valdez LM, La Rosa M. Severe COVID-19 during pregnancy and possible vertical transmission. Am J Perinatol 2020;37(08):861-865
33 Hu X, Gao J, Luo X, et al. Severe acute respiratory syndrome transmission in neonates born to mothers with coronavirus disease 2019. Obstet Gynecol 2020;136:1-3

34 Kuhrt K, McMicking J, Nanda S, Nelson-Piercy C, Shennan A. Placental abruption in a twin pregnancy at 32 week's gestation complicated by COVID-19, without vertical transmission to the babies. Am J Obstet Gynecol MFM 2020 (e-pub ahead of print). Doi: $10.1016 / j . a j o g m f .2020 .100135$

35 Lu D, Sang L, Du S, Li T, Chang Y, Yang X-A. Asymptomatic COVID19 infection in late pregnancy indicated no vertical transmission. J Med Virol 2020 (e-pub ahead of print). Doi: 10.1002/ jmv.25927

36 Mehta H, Ivanovic S, Cronin A, et al. Novel coronavirus-related acute respiratory distress syndrome in a patient with twin pregnancy: a case report. Case Rep Womens Health 2020;27:e00220

37 Piersigilli F, Carkeek K, Hocq C, et al. COVID-19 in a 26-week preterm neonate. Lancet Child Adolesc Health 2020;4(06): $476-478$

38 Sun M, Xu G, Yang Y, et al. Evidence of mother-newborn infection with COVID-19. Br J Anaesth 2020 (e-pub ahead of print). Doi: 10.1016/j.bja.2020.04.066

39 Xiong X, Wei H, Zhang Z, et al. Vaginal delivery report of a healthy neonate born to a convalescent mother with COVID-19. J Med Virol 2020;1-3. DOI: 10.1002/jmv.25857

40 Yan J, Guo J, Fan C, et al. Coronavirus disease 2019 in pregnant women: a report based on 116 cases. Am J Obstet Gynecol 2020 (e-pub ahead of print). Doi: 10.1016/j.ajog.2020.04.014

41 Yang P, Wang X, Liu P, et al. Clinical characteristics and risk assessment of newborns born to mothers with COVID-19. J Clin Virol 2020;127:104356

42 Zeng L, Xia S, Yuan W, et al. Neonatal early-onset infection with SARS-CoV-2 in 33 neonates born to mothers with COVID-19 in Wuhan, China. JAMA Pediatr 2020 (e-pub ahead of print). Doi: $10.1001 /$ jamapediatrics.2020.0878 